Tourism in Analysis

DOI: http://dx.doi.org/10.11606/issn.1984-4867.v27i3p524-542

\title{
Hospitalidade e Comunicação: a experiência de compras de serviços de viagens on-line
}

\author{
Hospitality and communication: the shopping \\ experience of online travel services \\ Hospitalidad y comunicación: la experiencia de compra \\ de servicios de viajes en Internet
}

\author{
Cintia Goldenberg ${ }^{1}$ \\ Rita de Cassia Pinotti ${ }^{2}$ \\ Hamilton $\mathrm{Pozo}^{3}$
}

\begin{abstract}
Resumo
O objetivo desta pesquisa é analisar de que forma a comunicação facilita a relação de hospitalidade entre uma empresa e seus clientes, com consequente geração de confiabilidade e vantagem competitiva. O método adotado foi exploratório de abordagem quali-quantitativa. A problemática trabalhada foi: a comunicação utilizada por uma empresa prestadora de serviços de viagens facilita a relação de hospitalidade com o atual e potencial cliente, com consequente vantagem competitiva? As hipóteses da pesquisa foram: $\mathrm{H} 1$ - A composição do website de uma empresa prestadora de serviços de viagens on-line contribui para a geração de confiabilidade e vantagem competitiva na prestação de serviços para seus clientes; e H2 - Uma comunicação adequada aos perfis de clientes atendidos pelas empresas estudadas contribui para o estabelecimento de uma relação de hospitalidade com os clientes da empresa. Como resultados, confirmou-se que a estrutura do website de uma empresa prestadora de serviços contribui para fortalecer a confiabilidade e a vantagem competitiva na prestação de serviços e, também, que a comunicação adequada aos perfis de clientes contribui para o fortalecimento de uma relação de hospitalidade com seus clientes.
\end{abstract}

Palavras-chave: Hospitalidade; Comunicação; Serviços; Confiabilidade.

\footnotetext{
${ }^{1}$ Mestre em Hospitalidade pela Universidade Anhembi Morumbi (UAM). São Paulo, São Paulo, Brasil. E-mail: cintiagoldenberg@hotmail.com

2 Mestre em Hospitalidade pela Universidade Anhembi Morumbi (UAM). Especialização em Administração pela Faculdade Getúlio Vargas (FGV). São Paulo, São Paulo, Brasil. São Paulo, São Paulo, Brasil. E-mail:rita_pinotti@yahoo.com.br

3 PhD em Business Administration pela California Coast University USA, Estados Unidos. Pós-Doutor em Administração pela Faculdade de Economia e Administração e Contabilidade da Universidade de São Paulo (FEA-USP). Mestre em Business Administration pelo Alexander Hamilton Institute USA, Estados Unidos. Docente e pesquisador do Programa de Mestrado e Doutorado em Hospitalidade da Universidade Anhembi-Morumbi (UAM). São Paulo, São Paulo, Brasil. E-mail:hprbrazil@hotmail.com
} 
Tourism in Analysis

\begin{abstract}
The objective of this research is to examine how communication facilitates the relationship of hospitality between a travel agency and its customers, with consequent generation of reliability and competitive advantage. The method used was a qualitative and quantitative exploratory approach. The problem was: communication used by a provider of travel services company facilitates the hospitality relationship with current and potential customers, with consequent competitive advantage? The hypotheses of the research were: H1 - The composition of the website of a company providing online travel services contributes to the generation of reliability and competitive advantage in providing services to their customers; and H2 - Proper communication to profiles customers served by companies studied contribute to the establishment of a hospitality connection with the company's customers. As a result, it was confirmed that the structure of the website of a company providing services contributes to strengthening the reliability and competitive advantage in providing services and also that adequate notice to customer profiles contributes to strengthening a relationship hospitality to its guests.
\end{abstract}

Keywords: Hospitality; Communication; Services; Reliability.

\title{
Resumen
}

El objetivo de esta investigación es examinar cómo la comunicación facilita la relación de hospitalidad entre una empresa y sus clientes, con la consiguiente generación de fiabilidad y ventaja competitiva. Se utilizó el método exploratorio con enfoque cualitativo y cuantitativo. El problema planteado fue el siguiente: ¿La comunicación utilizada por un proveedor de una empresa de servicios de viajes facilita la relación de hospitalidad con los actuales y potenciales clientes, con la consiguiente ventaja competitiva? Las hipótesis de la investigación fueron: (H1) - La existencia de una página web de una empresa de prestación de viajes en Internet contribuye a la generación de fiabilidad y ventaja competitiva en la prestación de servicios a sus clientes, y (H2) - La comunicación apropiada a los perfiles de clientes atendidos por las empresas estudiadas contribuye al establecimiento de una conexión de hospitalidad con los clientes de la compañía. Como resultado, se confirmó que la estructura de la página web de una empresa de prestación de servicios refuerza la fiabilidad y la ventaja competitiva en la prestación de servicios, así como la comunicación adecuada a los perfiles de los clientes fortalece la relación hospitalidad con sus huéspedes.

Palabras clave: Hospitalidad; Comunicación; Servicios; Fiabilidad.

\section{Introdução}

Após a virada do milênio, com a consolidação da sociedade da informação, o impulso da tecnologia e a disseminação das redes sociais, o comércio eletrônico ampliou sua escalada ascendente. Atualmente, vários tipos de negócios migraram para a web, tais como moda, 
entretenimento, varejo de eletrodomésticos e eletroeletrônicos - e o turismo não poderia ser exceção. A forma atual da Internet, particularmente a World Wide Web, tem sido uma ferramenta cada vez mais utilizada pelo setor de viagens. Porém, a literatura que examina Internet e turismo só recentemente passou a ser de interesse do setor e, rapidamente, se tornou um tema cada vez mais pesquisado.

O turismo on-line movimentou em 2012, aproximadamente, R\$ 13 bilhões no Brasil, registrando um crescimento de 20\% em relação ao ano anterior (TRANSAÇÕES..., 2015). Ratificando os números de mercado, o recente levantamento TravelClick North American Distribution Review, feito pela empresa americana de soluções de e-Commerce sobre o desempenho do setor de hospitalidade no primeiro trimestre de 2014, apontou que as Online Travel Agencies (OTA, ou Agências de Viagem On-line) registraram um crescimento de $9,2 \%$ em suas reservas se comparado ao volume obtido no mesmo período do ano anterior. Os pernoites comprados diretamente nas páginas dos hotéis tiveram um incremento de 6,1\% em relação a 2013, enquanto os Global Distribution Systems (GDS, ou Sistemas de Distribuição Global), usados pelas agências de viagens, tiveram o menor crescimento: 2,9\%. (TRAVELCLICK,2015). Esses dados mostram que o uso da Internet vem sendo o grande aliado do turista moderno, permitindo, como bem observado por Otto (2011), realizar múltiplas pesquisas, ver e fazer comentários on-line, buscar informações sobre destino e comparar tarifas. Permite ainda a integração com as redes sociais, promovendo a interação social.

Com base no exposto, este artigo tem como objetivo geral entender de que forma a comunicação facilita a relação de hospitalidade que ocorre entre uma empresa e seus clientes, com consequente geração de confiabilidade e vantagem competitiva. A questão levantada pela pesquisa é, portanto: a comunicação utilizada por uma empresa prestadora de serviços de viagens facilita a relação de hospitalidade com o atual e potencial cliente, com consequente vantagem competitiva?

Para o desenvolvimento da pesquisa, foram definidas duas hipóteses, sendo:

H1 - A composição do website de uma empresa prestadora de serviços de viagens on-line contribui para a geração de confiabilidade e vantagem competitiva na prestação de serviços para seus clientes;

H2 - Uma comunicação adequada aos perfis de clientes atendidos pelas empresas estudadas contribui para o estabelecimento de uma relação de hospitalidade com os clientes da empresa. 
O método adotado foi exploratório de abordagem quali-quantitativa, e para a coleta de dados foram utilizadas 22 questões fechadas, sendo 5 para classificação demográfica e 17 referentes à percepção dos respondentes com relação aos websites utilizados para aquisição de serviços de viagem para elas, utilizando escala Likert com notas de 1 a 5. Também foram utilizadas duas questões abertas cujas respostas permitiram a análise qualitativa do conteúdo. Como resultados, ratificou-se que a composição do website de uma empresa prestadora de serviços contribui para a geração da confiabilidade e da vantagem competitiva na prestação de serviços e que a comunicação adequada aos perfis de clientes atendidos pelas empresas estudadas contribui para o estabelecimento de uma relação de hospitalidade com os clientes da empresa.

\section{Referencial teórico}

\subsection{Hospitalidade e compras on-line}

A hospitalidade pode ser entendida como a relação que ocorre entre hóspede e anfitrião, que pode ser transposta a qualquer relação entre duas pessoas, entre pessoa e empresa e entre empresa e empresa. Para Lashley e Morrison (2004), é fundamental o relacionamento construído entre anfitrião e hóspede (ou empresa e cliente):

$\mathrm{O}$ entendimento mais amplo a respeito da hospitalidade sugere, em primeiro lugar, que esta é, fundamentalmente, o relacionamento construído entre anfitrião e hóspede. Para ser eficaz, é preciso que o hóspede sinta que o anfitrião está sendo hospitaleiro por sentimentos de generosidade, pelo desejo de agradar e por ver a ele, hóspede, enquanto indivíduo. (LASHLEY; MORRISON, 2004, p. 21)

Mesmo na relação comercial, o desejo de agradar, acolher e estar com o outro pode ser considerado como hospitalidade. Nesse contexto, essa relação está diretamente ligada à percepção da excelência do serviço prestado ao cliente e muitas vezes também na relação social que poderá ser estreitada.

Lashley e Morrison (2004) tratam a hospitalidade sob a perspectiva de três domínios, sendo eles o social, o privado e o comercial. O primeiro domínio estuda as relações de hospitalidade 
que ocorrem no cenário social, ou seja, é o trato com o outro desconhecido e as relações de mutualidade existentes. Já o segundo, o domínio privado, discute questões sobre o lar e o papel do anfitrião. Finalmente o terceiro, o comercial, trata a hospitalidade como parte da atividade econômica em nossa sociedade.

as preocupações atuais são, em parte, estabelecer uma rigorosa compreensão da amplitude do significado das atividades relacionadas com a hospitalidade, de modo que se possa entender melhor sua aplicação comercial. (LASHLEY; MORRISON, 2004, p.18)

Ainda segundo os autores, a intercessão dos três domínios (privado, social e comercial) citados anteriormente formam a experiência relativa à hospitalidade, e sua gestão executada pelas empresas é a que pode gerar vantagem competitiva.

No ambiente virtual, a relação de hospitalidade pode ou não se dar entre o website da empresa como anfitrião, que deve atender às expectativas de seu público-alvo e o internauta-usuário, como “convidado". Aqui se busca respaldo em Gotman (apud SOARES, 2013, p. 218-220), para quem a hospitalidade é "um processo de agregação do outro à comunidade e a inospitalidade é o processo inverso. [...] [que deve] carregar traços de acolhimento que se traduzam num serviço hospitaleiro".

Krug (2006) afirma que a arquitetura de design "interativa” considera o usuário como participante ativo e para isso deve estar atenta não apenas à comunicação, mas também aos elementos que garantam a acessibilidade (forma de apresentação e navegabilidade), usabilidade (facilidade para identificar e compreender o que se busca) e a visão dinâmica (páginas que sejam vistas e não lidas). As premissas do autor destacam a natureza hospitaleira mesmo no ambiente virtual, onde a interação humana é minimizada e deve ser muito bem observada, principalmente quando se trata da primeira experiência de compra de viagem.

A comunicação tem como base a relação que ocorre entre um emissor e um receptor de uma mensagem, que por sua vez é disseminada por canais que, por meio da evolução tecnológica, sofreram diversas alterações significativas até os dias atuais. Conforme apresentam Verma, Stock e McCarthy (2012), a maioria dos viajantes, ao buscar informação para uma estadia em determinado hotel, segue primeiro a recomendação de amigos e colegas, depois liga para essas fontes on-line (como o site da marca, OTAs e TripAdvisor), e, por fim, faz as reservas 
on-line. Os consumidores parecem ser mais influenciados por informações negativas no início - especialmente quando o conjunto global de comentários é negativo -, enquanto informações positivas e dados numéricos e detalhes de rating elevados possibilitam o aumento das intenções de reserva, de acordo com Sparks e Browning (2011). No entanto, uma vez que a procura não leva automaticamente à reserva, a fonte de informações não é completada. O relacionamento entre emissor e receptor, que pode ser transposto a empresa e cliente, vendedor e consumidor, também sofreu transformações devido às mudanças tecnológicas. A comunicação deixou de ser face a face e alcançou distâncias muito maiores, obtendo velocidade na produção e recepção da mensagem (BRANDÃO, 2011).

A comunicação se tornou mais pessoal ao conhecer mais claramente o perfil do consumidor, graças à tecnologia da informação (PIRES, 2010). O relacionamento com clientes é, atualmente, uma das maiores preocupações das empresas, pois é ele que gera a lealdade, retenção, melhora nas compras - e a Internet é um importante canal para o estabelecimento do vínculo empresa-cliente. As compras on-line e off-line têm algumas características que tornam cada uma delas mais adequada para um tipo de consumidor e, até mesmo para quem compra de ambas as maneiras, de acordo com as circunstâncias (ARCE-URRIZA; CEBOLLADA, 2013).

Como afirmam Biz e Lohmann (2005), a Internet eliminou parte da barreira do tempo, custo e distância, tornando a informação disponível e a comunicação mais rápida e interativa. Também, Remondes, Serrano e Mena (2015) concordam com a redução dos custos e tempos de resposta nas empresas do setor do turismo e enfatizam as mudanças que as novas tecnologias de informação e comunicação refletem na operacionalização, distribuição e estruturação da indústria do turismo; com destaque para os infomediários, empresas do tipo ".com" constituídas de uma avançada plataforma tecnológica e maciços investimentos em marketing que atualmente respondem pela desintermediação do setor.

A Internet propicia mais informação e vem se consolidando como um canal de vendas, o que exige respostas em tempo real à interatividade (COSTA, 2008) sendo que, para o consumidor, uma das vantagens do comércio eletrônico é a possibilidade de aquisição de produtos ou serviços a um preço mais competitivo.

Baseado na afirmação de Garai e Saratzaga (2012) em que o consumidor compra essencialmente informação, Bailey (2011) ressalta que o marketing on-line não depende apenas de 
sua qualidade na construção e design, mas também de uma gama de fatores interligados que proporcionam melhores experiências de compras à medida que compreendem melhor as preferências do cliente/visitante.

A gestão da comunicação em websites deve considerar os fatores que corroboram com comunicação a fim de facilitar a relação de hospitalidade que ocorre entre uma empresa com seus clientes, posto que viagens e hospedagem são dois dos principais produtos mais adquiridos através do comércio eletrônico (REMONDES; SERRANO; MENA, 2015).

Durante anos os acadêmicos têm debatido a importância de sites de hotéis, como pontos focais de uma ação de marketing e estratégia de vendas. Recentemente, parte da discussão acadêmica foi, principalmente, transferida para dois temas: em primeiro lugar, o uso dos meios de comunicação social para engajar potenciais consumidores e influenciar o comportamento de compra; e, em segundo lugar, a utilização eficaz dos agentes de viagens on-line (LEE; GUILLET; LAW, 2013). Sob a ótica da hospitalidade, este estudo questiona de que forma a comunicação facilita a relação de hospitalidade entre as empresas estudadas e seus clientes por meio de seus websites. No que diz respeito à influência da identidade social e de compra, alguns estudos de Wang e Chang, (2013) têm estabelecido que a influência social sobre a rede de sites sociais pode ter impacto na decisão de compra do cliente, incluindo comportamento de compra em mundos virtuais.

Enquanto os viajantes estão cada vez mais se voltando para a Internet para obter informações, muitos ainda estão hesitantes em fazer reservas on-line por causa da percepção de falta de um método de pagamento seguro, a falta de confiança na tecnologia e uma necessidade de um serviço personalizado e interface homem (WONG; LAW, 2005). Os viajantes preferem reservar suas viagens internacionais a partir de um agente de viagens em vez de reservá-las on-line, devido às vantagens das agências de viagens na interação social, experiência e a possibilidade de poupar tempo em pesquisa - embora eles acreditem que seja mais barato reservar on-line (BOGDANOVYCH et al., 2006).

Os determinantes de reservas de viagens on-line validados pelos pesquisadores são principalmente três vezes maiores, visto que, em primeiro lugar, a intenção de reserva on-line é determinada pelas características derivadas a partir da Internet, incluindo o risco percebido, a segurança, a conveniência e a economia de tempo, conforme exposto por Magnini e Karande (2011). Em segundo lugar, a decisão de reserva on-line é influenciada pelas características da linha de fornecedores, incluindo a qualidade da informação, preço 
competitivo, os padrões de confiabilidade, e o desempenho do serviço e sua reputação (KIM; KIN; SHIN, 2009; STEINBAUER; WERTHNER, 2007).

\subsection{Serviços e competitividade}

A oferta de serviços turísticos on-line divide dois conceitos básicos do marketing de serviços: intangibilidade (não podem ser vistos, tocados ou experimentados antecipadamente) e inseparabilidade (prestação de serviço e consumo simultâneos). Entretanto, a pesquisa do marketing de serviços evolui e Martin (1999) faz outras duas importantes considerações: a satisfação do cliente é uma função de sua expectativa e percepção, ambas influenciadas pelo prestador e a diferença entre concorrentes não é preço, mas o que o cliente percebe que recebeu em troca de seu dinheiro - o valor.

Do ponto de vista do cliente, o fato de algumas empresas manterem lojas físicas cria evidências tangíveis e soma valor à experiência de compra de serviços turísticos promovendo a mesma relação de hospitalidade para os clientes que preferem ainda o atendimento de forma tradicional. Isso valida a afirmação de Fitzsimmons e Fitzsimmons (2005) de que a elaboração de um sistema de prestação de serviços é um processo criativo, que se inicia por meio do conceito de serviço e perpassa por uma estratégia que seja dotada de características que possam distingui-lo da concorrência.

A criação de valor é inerente à vantagem competitiva. Wada e Moretti (2014) defendem a vantagem competitiva como parte do conjunto de situações que permite a determinada organização concorrer em seu negócio. As bases para ela ocorrer são múltiplas: preço, distribuição, qualidade e tecnologia, entre outros. Quanto mais camadas, maior a vantagem (HAMEL; PRAHALAD, 1995). Assim, pode-se relacionar o sucesso de uma empresa com sua VC e, portanto, com a capacidade de criação de valor. A vantagem competitiva de uma empresa é intrínseca a duas capacidades críticas: capacidade para sentir o mercado (e suas mudanças) e capacidade de ligação com o cliente, habilidades que permitam estreitar o relacionamento (DAY, 1994).

Ao encontro desses preceitos, Kotler (2000, p. 33) explica que a administração do marketing é "a ciência da escolha de mercados-alvo e da captação, manutenção e fidelização de cliente por meio da criação, da entrega e da comunicação de um valor superior para um cliente". O autor define valor como sendo "a razão entre o que o cliente recebe e o que ele dá". 
Martins (2015) sinaliza que uma alternativa para agregar valor ao processo de compra na loja virtual é oferecer, além de produtos de qualidade, motivos que convençam o consumidor a comprar, como simplicidade no processo de compra, suprimentos de necessidades reais do consumidor, personalização do marketing e identificação com o cliente, comunicação direta e resposta eficaz às interações.

Um elemento importante é que os grupos mais velhos têm maior aumento na probabilidade de informações de viagem e no comportamento de busca do que geração Y em geral, que já está acostumada ao mundo virtual, conforme afirmam Bronner e Hoog (2011). Existem diferenças substanciais de gênero, tanto em termos de atitudes para canais de informação quanto em preferências de funcionalidade do site de viagens.

Apesar de os viajantes estarem usando cada vez mais a internet, alguns preferem reservar suas viagens internacionais a partir de um agente de viagens em vez de reservá-las on-line, devido às vantagens das agências de viagens na interação social, experiência e a possibilidade de poupar tempo em pesquisa, embora eles acreditam que é mais barato reservar on-line (ESCOBAR-RODRIGUEZ; CARVAJAL-TRUJILLO, 2013).

Kim e Mauborgne (2005) e Pozo (2015) também sugerem examinar grupos estratégicos dentro do mesmo setor e ressaltam a importância de se compreender os fatores que influenciam as decisões dos clientes de optar por um entre dois critérios: preço e desempenho. Não é simplesmente conquistar novos segmentos e reduzir custos, mas, a partir dessa análise, é possível "formatar" o produto desenvolvendo uma matriz de valor de acordo com o perfil em potencial.

\section{Procedimentos metodológicos}

O objetivo desta pesquisa é entender de que forma a comunicação facilita a relação de hospitalidade que ocorre entre uma empresa e seus clientes, com consequente geração de confiabilidade e de vantagem competitiva. O método adotado foi exploratório de abordagem quali-quantitativa, sendo uma pesquisa empírica que é baseada em um método de amostragem

não probabilística. É, por conseguinte, uma amostra de conveniência. Especificamente, o conjunto de dados foi coletado em novembro de 2015 a partir de uma amostra de questionários on-line enviadas para cem pessoas. 
Esta pesquisa foi realizada com consumidores do setor de viagem com o intuito de entender apercepção do público em geral sobre a utilização de serviços de viagens on-line e sua futura intenção de reservar serviço de viagem pelo mesmo sistema. Na pesquisa foi desenvolvido um questionário para colher dados empíricos e na coleta de dados foram utilizadas 22 questões fechadas, sendo 5 para classificação demográfica e 17 referentes à percepção dos respondentes com relação aos websites utilizados para aquisição de serviços de viagem para elas. Utilizou-se a escala Likert com notas de 1 a 5, além de duas questões abertas cujas respostas permitiram a análise qualitativa do conteúdo. Os questionários foram enviados via e-mail, sem identificação dos respondentes utilizando-se um formulário em Excel para uma amostra de cem consumidores, obtendo-se um retorno de 44 questionários válidos. Foi realizada tabulação em Excel e análise pelo software SPSS 23.0 que é utilizado para análises estatísticas. A análise discriminante é mais apropriada quando a variável dependente não é métrica.

A amostra foi do tipo não probabilística e, segundo Vergara (2006), esse tipo de amostra se dá pela facilidade de acesso. Os questionários foram enviados a profissionais que tivessem já realizado compra em site de viagens. O período de coleta foi de 9 a 20 de novembro de 2015 . Após o fechamento do período de pesquisa foi realizada a tabulação e processamento de dados no Microsoft Excel para elaboração da análise estatística descritiva e posteriormente analisado pelo software SPSS 23.0.

\section{Análise dos dados}

No comércio do turismo on-line o consumidor compra essencialmente informação, e, como ressaltado por Bailey (2011), o marketing on-line não depende apenas de sua qualidade na construção e design, mas de uma gama de fatores interligados que proporcionam melhores experiências de compras à medida que compreendem melhor as preferências do cliente/visitante. Diante do exposto, este estudo exploratório tem como foco entender de que forma a comunicação facilita a relação de hospitalidade que ocorre entre uma empresa e seus clientes, com consequente geração de confiabilidade e vantagem competitiva. Para elaboração da análise as variáveis da pesquisa foram abordadas com foco em quatro dimensões: comunicação, confiabilidade, facilidade de compra e experiência de compra on-line. 
Tourism in Analysis

O perfil dos respondentes foi composto por $73 \%$ do sexo feminino e $27 \%$ do sexo masculino. Parte significativa da amostra (66\%) tem idade entre 31 e 50 anos. Em relação à escolaridade, $61 \%$ dos participantes têm pós-graduação. Referente à renda, 77\% dos respondentes possuem renda domiciliar superior a oito mil reais.

Gráfico 1 - Faixa etária dos respondentes

Faixa etária

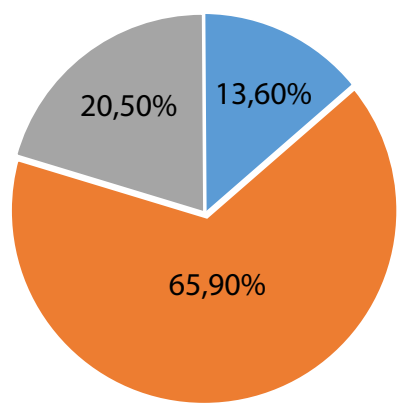

- 21 a 30 anos - 31 a 50 anos - mais de 50 anos

Fonte: Dados da pesquisa

Gráfico 2 - Escolaridade dos respondentes

Escolaridade

$4,50 \%$

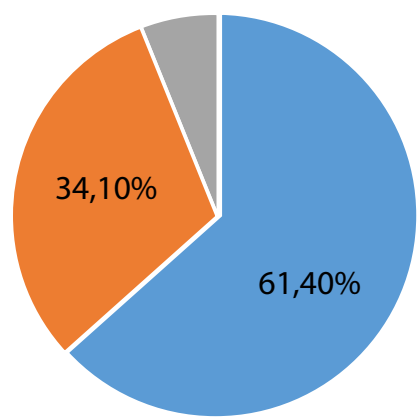

- Pós Graduação | Gradução — Ensino Médio

Fonte: Dados da pesquisa

A seguir são apresentados os dados mais expressivos verificados na etapa quantitativa pesquisa. Na Tabela 1 estão apresentados os dados de variáveis, média, desvio padrão e coeficiente de variância da análise quantitativa obtida pelo SPSS. 
Tourism in Analysis

Tabela 1 - Média, desvio padrão, coeficiente de variância

\begin{tabular}{|c|c|c|c|c|}
\hline \multicolumn{4}{|c|}{ Estatística de item } & \multirow{2}{*}{$\begin{array}{l}\mathrm{Cv} \\
\%\end{array}$} \\
\hline Quest. & VARIÁVEIS & MÉDIA & DESVIO PADRÃo & \\
\hline V1 & Suporte de compra & 4,75 & 0,43802 & 9,3 \\
\hline V2 & Organização/Informação & 4,886 & 0,32104 & 6,5 \\
\hline V3 & Apresentação visual & 3,866 & 0,84126 & 21,9 \\
\hline $\mathrm{V} 4$ & Compreensão & 4,296 & 0,6317 & 14,6 \\
\hline V5 & Informações claras & 4,864 & 0,34714 & 7,1 \\
\hline V6 & Opções de compra & 4,136 & 0,8238 & 19,5 \\
\hline V7 & Forma de pagamento & 4,341 & 0,86113 & 20,0 \\
\hline V8 & Navegabilidade & 1,682 & 0,85651 & 50,5 \\
\hline V9 & Preferência de compra & 3,682 & 1,15683 & 31,3 \\
\hline V10 & Redes sociais atualizadas & 3,818 & 0,78571 & 20,5 \\
\hline V11 & Conteúdo dinâmico & 2,932 & 0,84627 & 29,0 \\
\hline V12 & Informações dos destinos & 4,205 & 0,76492 & 18,4 \\
\hline V13 & Anúncio na mídia & 3,25 & 0,99124 & 30,8 \\
\hline V14 & Acesso a promoções & 3,114 & 0,99327 & 32,3 \\
\hline V15 & Políticas claras & 4,773 & 0,47562 & 9,8 \\
\hline V16 & Login por redes sociais & 2,955 & 0,88802 & 29,7 \\
\hline V17 & Experiência em compras & 3,591 & 0,8712 & 24,2 \\
\hline
\end{tabular}

Fonte: Dados da pesquisa

Analisando os dados da Tabela1, tem-se que as variáveis "Apresentação visual", "Forma de pagamento", "Preferência de compra", "Redes sociais atualizadas", "Conteúdo dinâmico", "Anúncio na mídia", "Acesso a promoções", "Login por redes sociais" e "Experiência em compras" apresentaram o coeficiente de variância maior que $20 \%$.

Na Tabela 2 estão apresentados os dados relativos à correlação entre as variáveis da pesquisa. Com relação aos dados de correlação entre as variáveis da amostra, temos: "Login por redes sociais" possui maior correlação com o item "Suporte de compras" $(0,45)$; "Informação do destino" é relacionada em maior grau com "Organização/informação" (0,67); o item “Compreensão" possui maior correlação com "Informação do destino" $(0,43)$; “Informação de compras" foi relacionado com a variável "Compreensão" $(0,57)$, bem como o item "Políticas claras" relacionado com "Informações claras" $(0,88)$; a variável "Redes sociais atualizadas", possui maior correlação com "Opções de compras" $(0,66)$; a correlação de "Forma de pagamento" com "Informação do destino" $(0,77)$ é forte; "Navegabilidade" tem correlação, em maior grau, com a variável "Acesso a promoções" $(0,60)$; "Preferência de compras" possui 0,60 de correlação com "Forma de pagamento"; a variável "Redes sociais atualizadas" está alinhada com a variável "Acesso a promoções”, com índice de correlação 


\begin{tabular}{|c|c|c|c|c|c|c|c|c|c|c|c|c|c|c|c|c|}
\hline 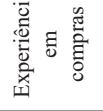 & $\mid \begin{array}{l}\overline{0} \\
0\end{array}$ & \begin{tabular}{|l|} 
\\
\\
0 \\
0 \\
0
\end{tabular} & $\begin{array}{l}0 \\
0 \\
0 \\
0\end{array}$ & $\begin{array}{l}0 \\
0 \\
0 \\
0\end{array}$ & $\begin{array}{l}\vdots \\
\vdots \\
\vdots\end{array}$ & 焉 & $\begin{array}{l}\text { a } \\
\text { and }\end{array}$ & \begin{tabular}{l|l}
0 \\
0 \\
0 \\
0 \\
0
\end{tabular} & : & $\stackrel{0}{0}$ & 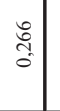 & $\overline{\frac{\pi}{0}}$ & & 党: & : & \\
\hline 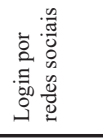 & 亭 & 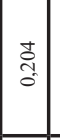 & 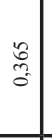 & $\begin{array}{l}\text { के } \\
\text { ते }\end{array}$ & : & $\stackrel{E}{0}$ & 总 & $\mid \begin{array}{l}\hat{a} \\
\vdots \\
0\end{array}$ & $\frac{\infty}{0}$ & 总 & $\begin{array}{l}\text { gे } \\
\text { g. } \\
0\end{array}$ & त్ & 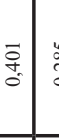 & ב. & & 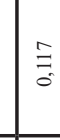 \\
\hline 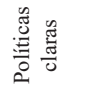 & $\begin{array}{l}\cong \\
0 \\
0\end{array}$ & \begin{tabular}{|c|}
$\infty$ \\
0 \\
0 \\
0
\end{tabular} & \begin{tabular}{l|}
0 \\
0 \\
0 \\
0
\end{tabular} & \begin{tabular}{l|l} 
& $\infty$ \\
0 \\
0 \\
0
\end{tabular} & 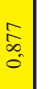 & 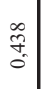 & 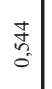 & \begin{tabular}{|l|}
0 \\
0 \\
0 \\
0
\end{tabular} & हृ. & $\begin{array}{l}\text { 守 } \\
\text { o }\end{array}$ & $\begin{array}{l}8 \\
0 \\
0\end{array}$ & 局 & đે. & $\frac{E}{0}$ & & $\begin{array}{l}0 \\
\infty \\
0 \\
0\end{array}$ \\
\hline 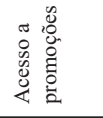 & $\mid \begin{array}{l}7 \\
0 \\
0 \\
i\end{array}$ & \begin{tabular}{|l|}
$\hat{n}$ \\
$\hat{c}$ \\
0
\end{tabular} & $\begin{array}{l}n \\
\vdots \\
0 \\
0 \\
1\end{array}$ & $\begin{array}{l}\exists \\
\vdots \\
0\end{array}$ & 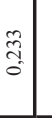 & ल⿸厂 & $\begin{array}{l}\text { I } \\
\text { a } \\
0\end{array}$ & $\mid$ & 嵩 & है & $\begin{array}{c}\infty \\
\text { के } \\
0 \\
0\end{array}$ & त्रे & 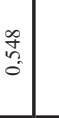 & & & $\frac{0}{0}$ \\
\hline 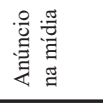 & 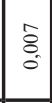 & \begin{tabular}{|l|} 
\\
âd \\
\end{tabular} & $\begin{array}{l}0 \\
\vdots \\
0\end{array}$ & $\begin{array}{l}8 \\
i \\
\end{array}$ & $\begin{array}{l}0 \\
\vdots \\
\vdots \\
\end{array}$ & : & $\begin{array}{l}0 \\
\vdots \\
0 \\
i\end{array}$ & $\begin{array}{l}0 \\
\text { : } \\
0\end{array}$ & 营 & \begin{tabular}{l}
$\infty$ \\
$\stackrel{\infty}{\infty}$ \\
\hdashline
\end{tabular} & 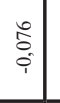 & $\begin{array}{l}\text { o } \\
: \\
0 \\
0\end{array}$ & & 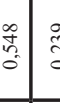 & & ĩ \\
\hline 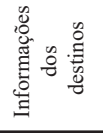 & 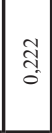 & $\mid$\begin{tabular}{|c|} 
\\
0 \\
0
\end{tabular} & $\begin{array}{l}3 \\
? \\
0 \\
0\end{array}$ & $\begin{array}{l}n \\
\tilde{a} \\
0 \\
0\end{array}$ & $\begin{array}{c}\hat{r} \\
\hat{0} \\
0\end{array}$ & वे. & $\begin{array}{l}1 \\
E \\
0\end{array}$ & 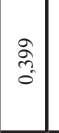 & 离 & $\begin{array}{l}0 \\
0 \\
0 \\
0\end{array}$ & $\begin{array}{l}\hat{A} \\
0 \\
0\end{array}$ & & 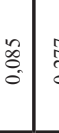 & \begin{tabular}{c|c} 
त्रे \\
co
\end{tabular} & & $\overline{\mathrm{I}}$ \\
\hline 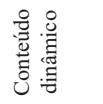 & $\begin{array}{l}\hat{a} \\
\hat{a} \\
0 \\
\end{array}$ & | & वे & वे & त् & 气े & 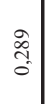 & $\mid$ & हี & $\begin{array}{l}\hat{n} \\
\hat{n} \\
0\end{array}$ & & त् & 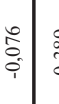 & 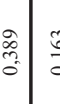 & & S. \\
\hline & \begin{tabular}{|c|}
$\infty$ \\
0 \\
0 \\
0
\end{tabular} & $\mid \begin{array}{l}0 \\
\substack{g \\
0 \\
0}\end{array}$ & 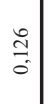 & $\begin{array}{l}5 \\
\vdots \\
0 \\
0\end{array}$ & $\begin{array}{l}0 \\
\vdots \\
0 \\
0\end{array}$ & : & $\frac{\infty}{\infty}$ & 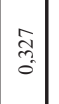 & $\frac{\vec{c}}{9}$ & & $\begin{array}{l}\tilde{0} \\
0 \\
0\end{array}$ & : & \begin{tabular}{l}
$\infty$ \\
$\infty$ \\
\hdashline \\
\hdashline
\end{tabular} & 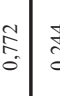 & & 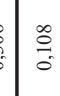 \\
\hline 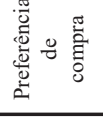 & \begin{tabular}{|l|}
$\infty$ \\
0 \\
0 \\
0 \\
0
\end{tabular} & \begin{tabular}{|l|}
2 \\
0 \\
0
\end{tabular} & $\begin{array}{l}1 \\
0 \\
0 \\
0\end{array}$ & \begin{tabular}{l|}
0 \\
0 \\
0 \\
0
\end{tabular} & $\begin{array}{l}5 \\
f \\
0 \\
0\end{array}$ & $\begin{array}{l}\text { bे } \\
\vdots \\
0 \\
0\end{array}$ & $\begin{array}{l}\tilde{0} \\
0 \\
0\end{array}$ & $\begin{array}{l}0 \\
0 \\
0 \\
0\end{array}$ & & $\begin{array}{l}\overrightarrow{0} \\
\bar{i}\end{array}$ & 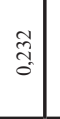 & 离 & 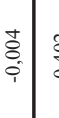 & 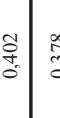 & & 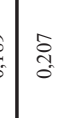 \\
\hline 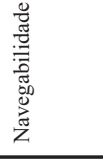 & $\begin{array}{l}0 \\
0 \\
0\end{array}$ & \begin{tabular}{|l|} 
\\
$f$ \\
0 \\
0
\end{tabular} & $\begin{array}{ll}0 \\
0 \\
0 \\
0 \\
0\end{array}$ & \begin{tabular}{l|}
$\vec{a}$ \\
$\vdots$ \\
0
\end{tabular} & 高 & $\begin{array}{l}\text { कo } \\
\text { i. }\end{array}$ & $\begin{array}{l}0 \\
0 \\
0 \\
0 \\
0\end{array}$ & & $\begin{array}{l}\text { लि } \\
\text { ?. }\end{array}$ & हू, & 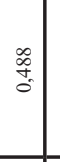 & बू. & 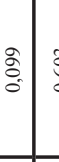 & בְ: & $\begin{array}{c}0 \\
\vdots \\
\vdots\end{array}$ & $\begin{array}{l}0 \\
0 \\
0 \\
0\end{array}$ \\
\hline 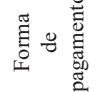 & \begin{tabular}{|l|}
$\stackrel{\infty}{0}$ \\
0
\end{tabular} & \begin{tabular}{|l|} 
\\
0 \\
0 \\
0 \\
0
\end{tabular} & $\begin{array}{l}\infty \\
\stackrel{\infty}{0} \\
0\end{array}$ & $\begin{array}{l}f \\
0\end{array}$ & $\begin{array}{l}0 \\
0 \\
0 \\
0 \\
0\end{array}$ & I্ & & \begin{tabular}{l|}
0 \\
0 \\
0 \\
0
\end{tabular} & : & $\stackrel{\infty}{0}$ & $\mid$ & E. & 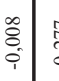 & 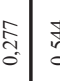 & & 志 \\
\hline 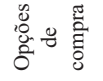 & $\begin{array}{l}\vec{m} \\
\hat{\sigma} \\
0\end{array}$ & \begin{tabular}{|l|} 
\\
$\dot{g}$ \\
0
\end{tabular} & $\begin{array}{l}0 \\
: \\
0 \\
0\end{array}$ & $\begin{array}{ll}0 \\
0 \\
0 \\
0\end{array}$ & $\frac{8}{0}$ & & $\begin{array}{l}7 \\
0 \\
0\end{array}$ & $\mid$\begin{tabular}{|c}
0 \\
$\dot{s}$ \\
$\dot{c}$ \\
$i$
\end{tabular} & 茴 & : & 竞 & तू & $\stackrel{\infty}{\infty}$ & 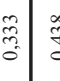 & & $=\begin{array}{l}\text { I } \\
\text { g }\end{array}$ \\
\hline 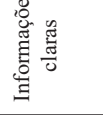 & $\begin{array}{l}\hat{g} \\
\text { ó } \\
0\end{array}$ & $\mid \begin{array}{l}\mid \frac{\alpha}{\circ} \\
\frac{\circ}{\circ}\end{array}$ & $\begin{array}{l}\text { के } \\
\text { के }\end{array}$ & $\begin{array}{l}\bar{z} \\
0\end{array}$ & & $\frac{m}{0}$ & $\begin{array}{l}\infty \\
\vdots \\
0 \\
0\end{array}$ & 范 & 点 & $\begin{array}{l}0 \\
\vdots \\
\vdots \\
0\end{array}$ & สี & 点 & $\begin{array}{l}\stackrel{0}{\Rightarrow} \\
i \\
i\end{array}$ & $\begin{array}{c}a \\
c \\
0\end{array}$ & $\begin{array}{ll}0 \\
\\
\end{array}$ & $\frac{0}{0}$ \\
\hline 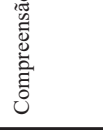 & \begin{tabular}{|c|c}
$\tilde{3}$ \\
0 \\
0
\end{tabular} & $\mid$\begin{tabular}{|c|}
0 \\
$\vdots$ \\
0 \\
0
\end{tabular} & \begin{tabular}{c|c}
$r$ \\
0 \\
0
\end{tabular} & & $\vec{z}$ & 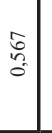 & $\begin{array}{l}5 \\
⿱ \\
0 \\
0\end{array}$ & $\mid$\begin{tabular}{|c}
$\vec{a}$ \\
0 \\
0
\end{tabular} & $\begin{array}{c}0 \\
0 \\
0 \\
0\end{array}$ & $\begin{array}{l}1 \\
\vdots \\
0\end{array}$ & ఫે & $\begin{array}{l}\text { 总 } \\
0 \\
0\end{array}$ & : & בa & 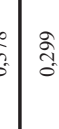 & : \\
\hline 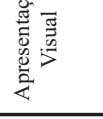 & $\begin{array}{l}0 \\
\vdots \\
0 \\
0\end{array}$ & $\left|\begin{array}{c|}\stackrel{0}{\vec{\lambda}} \\
0 \\
0\end{array}\right|$ & & $\begin{array}{l}\vec{r} \\
0 \\
0\end{array}$ & $\begin{array}{l}\text { के } \\
\partial_{0}^{\circ}\end{array}$ & : & $\begin{array}{l}\infty \\
\frac{\infty}{0} \\
0\end{array}$ & $\mid \begin{array}{l}0 \\
0 \\
0 \\
0 \\
0\end{array}$ & 蒫 & 용 & ते & 急 & : & 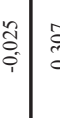 & : & ${ }_{0}^{\infty}$ \\
\hline 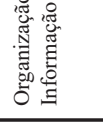 & $\begin{array}{l}\pi \\
2 \\
0\end{array}$ & & 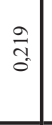 & \begin{tabular}{l|}
$\infty$ \\
0 \\
0 \\
0 \\
0
\end{tabular} & $\begin{array}{l}\infty \\
\stackrel{\infty}{0}\end{array}$ & बे & 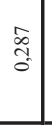 & $\begin{array}{l}\text { F } \\
\text { f. }\end{array}$ & $\stackrel{\frac{m}{2}}{0}$ & $\begin{array}{l}0 \\
\dot{g} \\
\dot{q} \\
0\end{array}$ & 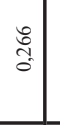 & है & 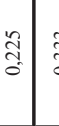 & 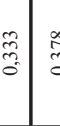 & : & 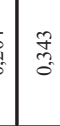 \\
\hline 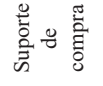 & & 党 & : & a & $\begin{array}{l}\vec{m} \\
\hat{0} \\
0\end{array}$ & 离 & $\frac{\mathrm{C}}{\circ}$ & $\mid$\begin{tabular}{|l}
$\mid$ \\
0 \\
0 \\
0 \\
0
\end{tabular} & $\begin{array}{l}\infty \\
\infty \\
b^{3}\end{array}$ & क्वे & I & 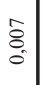 & : & 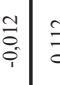 & & $\frac{\mathrm{g}}{0}$ \\
\hline & 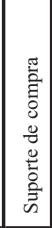 & 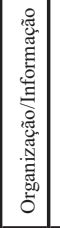 & 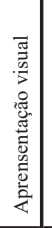 & 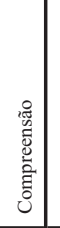 & 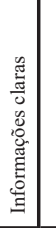 & 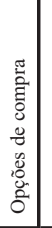 & 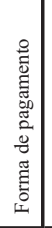 & 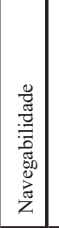 & 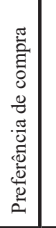 & 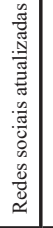 & 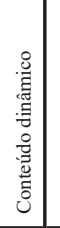 & 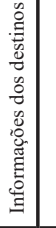 & | & 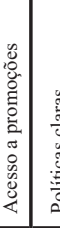 & 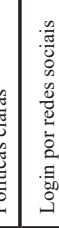 & 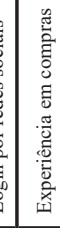 \\
\hline
\end{tabular}


de 0,77 ; "Informação de destino" teve correlações com “Opções de compras" $(0,77)$; o item "Anúncio na mídia" está ligado por correlação de 0,55 com a variável "Acesso à informação"; "Experiência de compras" está correlacionada com "Navegabilidade" $(0,37)$.

As variáveis com maior média e menor desvio padrão foram "Organização/Informação", "Informações claras" e "Políticas claras" (média: 4,89; 4,86 e 4,77, respectivamente; desvio padrão: 0,32; 0,35 e 0,48, respectivamente). Em contrapartida, a variável "Navegabilidade" foi a que obteve a menor média $(1,68)$. As variáveis que apresentaram maior índice de correlação foram: "Políticas claras" e "Informações claras".

O alfa de Cronbach das questões relativas às dezessete variáveis foi de 0,672 , valor considerado abaixo do ideal de 0,70 - o que indica a recomendação de continuidade do estudo com maior número de questões.

Tabela 3 - Alfa de Cronbach

\begin{tabular}{c|c|c}
\hline Alfa de Cronbach & Alfa de Cronbach com base em itens padronizados & Número de itens \\
\hline 0,672 & 0,696 & 17 \\
\hline
\end{tabular}

Fonte: Dados da pesquisa

Do ponto de vista qualitativo, as respostas às perguntas abertas: "Qual a vantagem percebida em se comprar via sites os serviços de viagem?" e "Tem algo que não tenha lhe agradado em alguma experiência de compra?", apresentaram homogeneidade nas repostas que indicam a praticidade e ganho de tempo como a principal vantagem percebida na compra de serviços de viagem on-line.

$\mathrm{Na}$ amostra foi encontrada correlação estatisticamente significante entre faixa etária e escolaridade em todas as dimensões desses dois conceitos. Contudo, apesar de estatisticamente significante, as correlações observadas não eram altas. O maior valor encontrado do coeficiente de Pearson foi de 0,522 (entre "Políticas claras" e a "Informações claras") e mostrou, também, que apenas 17\% de comportamento compartilhado há entre os dois construtos. A regressão linear mostrou um resultado positivo, acima de $42 \%$.

Em relação à experiência de compras, poucas respostas relatam problemas, sendo que estes se relacionavam a problemas com o pagamento e a finalização da compra, causando insegurança no usuário. Os aspectos da comunicação mais relevantes, conforme apontados pela pesquisa, estão relacionados com a clareza das informações, mais do que com o aspecto 
visual e estético do website. Essas informações incluem aspectos técnicos sobre reservas e compras, clareza nas políticas de reservas, compras e cancelamento.

A dimensão "confiabilidade" se mostra evidente nas variáveis (V15) e (V5), ambas relativas à clareza na comunicação sobre as políticas de compras, reservas e cancelamentos e nas informações técnicas disponibilizadas nos websites. Uma comunicação efetiva permite que essas informações se apresentem de forma clara, objetiva e completa, transmitindo confiança ao usuário do website.

Outra dimensão que se mostrou relevante foi a facilidade de compra, que aparece tanto na parte quantitativa como na parte qualitativa. Apesar de não estar diretamente relacionada a aspectos diretos de comunicação (linguagem e comunicação visual), essa dimensão é facilitada por uma comunicação eficiente.

Portanto, a pesquisa confirma que a Internet eliminou parte da barreira do tempo, custo e distância, tornando a informação disponível e a comunicação mais rápida e interativa, como afirmam (BIZ; LOHMANN, 2005).

Um website que se mostre confiável e cujos processos de compra, reserva, pagamento e cancelamentos sejam simples ao ponto de serem intuitivos facilita a relação de hospitalidade que ocorre entre uma empresa e seus clientes, com consequente geração de confiabilidade e vantagem competitiva. A relação de hospitalidade, entendida como o processo de agregação do outro à comunidade (ou ambiente, ou empresa, ou mesmo website), pode ou não carregar traços de acolhimento que se traduzem num serviço hospitaleiro de acordo com Soares, (2013), e um website "hospitaleiro" facilita o acolhimento do visitante.

\section{Considerações finais}

Esta pesquisa teve por objetivo entender de que forma a comunicação facilita a relação de hospitalidade que ocorre entre uma empresa e seus clientes, com consequente geração de confiabilidade e vantagem competitiva. Entretanto, como esperado, os resultados revelam que a prestação de serviços de viagens on-line tem sido uma tendência crescente, mas as agências de viagens ainda mantêm o canal de serviço de viagem não virtual como principal meio de reservas e vendas de viagens, uma vez que agência de viagens ainda é o canal 
preferido para os clientes obterem informações de viagem - e boa parte dos inquiridos ainda depende de agência de viagens para sua futura viagem.

As hipóteses da pesquisa H1 (a composição do website de uma empresa prestadora de serviços de viagens on-line contribui para a geração de confiabilidade e vantagem competitiva na prestação de serviços para seus clientes) e H2 (uma comunicação adequada aos perfis de clientes atendidos pelas empresas estudadas contribui para o estabelecimento de uma relação de hospitalidade com os clientes da empresa) foram ratificadas. Portanto, não há uma relação clara entre essas variáveis.

Foi possível inferir que a vantagem competitiva é gerada quando a comunicação é adequada ao perfil do cliente. O perfil socioeconômico e escolar da maior parte da amostra enquadra-se no estrato superior das classes sociais da cidade de São Paulo. Não houve referências à dificuldade de compreensão do conteúdo dos websites, mas apontamentos relativos à clareza de informações necessárias para efetivar as compras de forma segura e confiável.

Devido à relevância dos temas abordados neste estudo, recomenda-se a continuidade dessa pesquisa a partir deste estudo exploratório. Uma vez que o alfa de Cronbach foi considerado abaixo do ideal de 0,70 , sugerindo-se a ampliação do número de variáveis para a continuidade do estudo, visto que outras pesquisas não encontraram relações significativas de influência social sobre a intenção de compra on-line (ESCOBAR-RODRIGUEZ; CARVAJAL-TRUJILLO, 2013).

Em resumo, os resultados suportam as seguintes conclusões. Em primeiro lugar, os sites de viagens on-line e sua prestação de serviços têm crescido como um canal de distribuição direta e popular no setor de viagens. Em segundo lugar, os clientes têm a intenção de reservar serviços de viagens on-line, embora ainda prefiram agências de viagens tradicionais. Embora haja argumentos bastante contraditórios na hipótese de desintermediação na indústria de viagens, isso implica que o fenômeno da desintermediação exista nesse setor, e os intermediadores são ainda importantes para que o fornecimento de serviços de viagens se mantenha competitivo. Este estudo ofereceu algumas informações valiosas sobre o serviço de vendas on-line de viagens. Uma série de limitações do estudo é reconhecida no processo para melhor interpretar os resultados. Em primeiro lugar, o estudo empírico foi realizado com um número pequeno de entrevistados, requerendo, no futuro, mais estudos empíricos sobre como avaliar a qualidade dos serviços de viagens on-line para atender às necessidades do cliente. Esse fato reforça a necessidade de mais estudos sobre este tema. 
Tourism in Analysis

\section{Referências}

ARCE-URRIZA, M.; CEBOLlADA, J. Determinants of channel choice: online vs off-line: An application to a grocery chain. Cuadernos de Economia y Direccion de la Empresa, v. 16, n. 2, p. 108-122, 2013.

BAILEY, M. Internet marketing. New Jersey: Wiley, 2011.

BIZ, A.; LOHMAN, G. A importância da internet para as Agências de Viagens Brasileiras utilizadoras do GDS Amadeus. Turismo e Desenvolvimento, Aveiro, v. 2, n. 2, p. 73-83, 2005.

BRANDÃO, V. C. Comunicação e marketing na era digital: a internet como mídia e canal de vendas. In: CONGRESSO BRASILEIRO DE CIÊNCIAS DA COMUNICAÇÃO, 24., 2011, Campo Grande. Anais...Campo Grande: Intercom, 2011.

BOGDANOVYCH, A. et al. Travel agents vs. online booking: tackling the shortcomings of nowadays online tourism portals. In: HITZ, M.; SIGALA, M.; MURPHY, J. (Eds.). Information and Communication Technologies in Tourism 2006. Vienna: Springer, 2006. p. 418-428.

BRONNER, F.; HOOG, R. D. Vacationers and eWOM: who posts, and why, where, and what? Journal of Travel Research, v. 50, n. 1, p. 15-26, 2011.

CÂMARA, E. O comércio eletrónico e o turismo. 2012. 90 f. Teses (Mestrado em Gestão) Universidade dos Açores, Açores, 2012.

COSTA, R. Introdução à gestão hoteleira. Lisboa; Porto: Lidel, 2008.

GRUPO DE MÍDIA SÃO PAULO. Mídia dados Brasil 2014. São Paulo: Grupo de Mídia São Paulo, 2014. Disponível em: <http://sunflower2.digitalpages.com.br/html/reader/119/38924>. Acesso em: 8 dez. 2016.

DAY, G. S. The capabilities of market-driven organizations. Journal of Marketing, New York, v. 58, n. 4, p. 37-52, 1994.

ESCOBAR-RODRIGUEZ, T.; CARVAJAL-TRUJILLO, E. Online drivers of consumer purchase of website airline tickets. Journal of Air Transport Management, v. 32, n. 1, p. 58-64, 2013.

FITZSIMMONS, J. A.; FITZSIMMONS, M. J. Administração de Serviços: operações, estratégia e tecnologia da informação. 4. ed. Porto Alegre: Bookman, 2005.

GARAI, G.; SARATZAGA, G. Nuevo paradigma turístico del siglo XXI: el ejemplo del turismo industrial. Journal of Tourism and Development, v. 1, p. 61-76, 2012.

GOTMAN, A. Le sens de l'hospitalité. Paris: Presses Universitaires de France, 2001. 
Tourism in Analysis

HAMEL, G.; PRAHALAD, C. K. Competindo pelo futuro. Rio de Janeiro: Campus, 1995.

KIM, H.; KIN, T.T.; SHIN, S.W. Modeling roles of subjective norms and eTrust in customers' acceptance of airline B2C eCommerce website. Tourism Management, v. 30, n. 2, p. 266-277, 2009.

KIM, W. C.; MAUBORGNE, R. A estratégia do oceano azul: como criar novos mercados e tronar a concorrência irrelevante. Rio de Janeiro: Elsevier, 2005.

KOTLER, P. Administração de marketing. São Paulo: Prentice Hall, 2000.

KRUG, S. Não me faça pensar. Rio de Janeiro: Alta Books, 2006.

LASHLEY, C.; MORRISSON, A. Em busca da hospitalidade-perspectiva para um mundo globalizado. Barueri: Manole,2004.

LEE, H.; GUILLET, B. D.; LAW, R. An examination of the relationship between online travel agents and hotels a case study of choice hotels international and Expedia.com. Cornell Hospitality Quarterly, v. 54, n. 1, p. 95-107, 2013.

MAGNINI, V. P.; KARANDE, K. Understanding consumer services buyers based upon their purchase channel. Journal of Business Research, v. 64, n. 6, p. 543-550, 2011.

MARTIN, C. The history, evolution and principles of services marketing: poised for the new millennium. Marketing Intelligence and Planing, v. 17, n. 7, p. 324-328, 1999.

MARTINS, F. Experiência é mais importante que preço e produto. E-commerce News, 25 mar. 2015. Disponível em: $<$ http://bit.ly/2fBPRoW>. Acesso em: 9 maio 2015.

OTTO, G. O QI digital do turismo. Hôtelier News, 18 maio 2011. Disponível em: < http://bit.ly/ 2foAx3V>. Acesso em: 27 nov. 2016.

PIRES, L. C. Análise dos impactos da tecnologia de informação e comunicação para o turismo. Revista Acadêmica Observatório de Inovação do Turismo, Rio de Janeiro, v. 4, p. 2-5, 2010.

POZO, H. Hospitality industries management: hospitality revenues in Brazil. Saarbrücken: Lambert Academic Publishing, 2015.

REMONDES, J.; SERRANO, V.; MENA, R. Marketing on-line, comércio eletrônico e hotelaria: estudo sobre a marcação de hotéis na internet em mercados business-to-business. Revista Iberoamericana de Turismo, Penedo, v. 5, n. 1, p. 114-130, 2015.

SOARES, C. M. P. Hospitalidade virtual: uma tentativa de compreensão. Revista Hospitalidade, São Paulo, v. 10, n. 2, p. 213-233, 2013. 
Tourism in Analysis

SPARKS, B. A.; BROWNING, V. The impact of online reviews on hotel booking intentions and perception of trust. Tourism Management, v. 32, n. 6, p. 1310-1323, 2011.

STEINBAUER, A.; WERTHNER, H. Consumer Behaviour in e-Tourism. Information and Communication Technologies in Tourism, v. 2, p. 65-76, 2007.

TRANSAÇÕES no e-commerce crescem 46\% em 2012, diz Braspag. E-commerce News, 11 dez. 2012. Disponível em: <http://bit.ly/2fBNYIQ>. Acesso em: 27 nov. 2016.

TRAVELCLICK. TravelClick North American Distribution Review. 2015.

VERGARA, S. C. Projetos e relatórios de pesquisa em administração. São Paulo: Atlas, 2006.

VERMA, R.; STOCK, D.; MCCARTHY, L. Customer preferences for online, social media, and mobile innovations in the hospitality industry. Cornell Hospitality Quarterly, v. 53, n. 2, p. 183-186, 2012.

WADA, E. K.; MORETTI, S. L. A. Hospitalidade: dos domínios às lentes e sua inserção nos processos de gestão. Caderno de Estudos e Pesquisas do Turismo, Curitiba, v. 3, p. 95-105, 2014.

WANG, J. C.; CHANG, C. H. How online social ties and product-related risks influence purchase intentions: a Facebook experiment. Electronic Commerce Research and Applications, v. 12, n. 5, p. 337-346, 2013.

WONG, J.; LAW, R. Analysing the intention to purchase on hotel websites: a study of travellers to Hong Kong. International Journal of Hospitality Management, v. 24, n. 2, p. 311-329, 2005.

Recebido em: 13/01/2016 (1ª versão) 11/08/16 ( $2^{\mathrm{a}}$ versão)

Aprovado em: 15/08/2016 